Gut and Liver, Vol. 12, No. 4, July 2018, pp. 457-462

\title{
Electrohydraulic Lithotripsy for Difficult Bile Duct Stones under Endoscopic Retrograde Cholangiopancreatography and Peroral Transluminal Cholangioscopy Guidance
}

Rieko Kamiyama, Takeshi Ogura, Atsushi Okuda, Akira Miyano, Nobu Nishioka, Miyuki Imanishi, Wataru Takagi, and Kazuhide Higuchi

Second Department of Internal Medicine, Osaka Medical College, Takatsuki, Japan

Background/Aims: Electrohydraulic lithotripsy (EHL) under endoscopic retrograde cholangiopancreatography (ERCP) guidance can be an option to treat difficult stones. Recently, a digital, single-operator cholangioscope (SPY-DS) has become available. Peroral transluminal cholangioscopy (PTLC) using SPY-DS has also been reported. In this retrospective study, the technical feasibility and clinical effectiveness of EHL for difficult bile duct stones under ERCP guidance and under PTLC guidance was examined. Methods: In this pilot study, patients with difficult bile duct stones between July 2016 and July 2017 were retrospectively enrolled. Results: Forty-two consecutive patients underwent EHL using a SPYDS; 34 patients underwent EHL under ERCP guidance, and the other 8 patients underwent EHL under PTLC guidance. Median procedure time was 31 minutes (range, 19 to 66 minutes). The median number of EHL sessions was 1 (range, 1 to 2), and that of ERCP sessions was also 1 (range, 1 to 3 ). The rate of complete stone clearance was 98\% (41/42). Adverse events such as cholangitis and acute pancreatitis were seen in 14\% (6/42), which could be treated conservatively. Conclusions: EHL using SPY-DS was technically feasible, not only under ERCP guidance, but also PTLC guidance. A prospective clinical study of EHL using SPY-DS is needed. (Gut Liver 2018;12:457-462)

Key Words: Endoscopic ultrasound intervention; Cholangiopancreatography, endoscopic retrograde; Common bile duct; Choledcholithiasis

\section{INTRODUCTION}

Bile duct stones are extremely common. They have a potential risk of acute cholangitis or pancreatitis, and they are usually treated under endoscopic retrograde cholangiopancreatography (ERCP) guidance. ${ }^{1-4}$ However, treating bile duct stones is sometimes challenging due to patient factor, such as surgically altered anatomy, a periampullary diverticulum, or a sigmoidshaped common bile duct (CBD), and stones factors, such as a large number or size of stones, intrahepatic bile duct stones, or bile duct stenosis. ${ }^{5}$

Electrohydraulic lithotripsy (EHL) under ERCP guidance can be an option to treat difficult stones. ${ }^{6-9}$ Recently, a digital single-operator cholangioscope (SPY-DS; SpyGlass DS, Boston Scientific, Marlborough, MA, USA) has become available. The SPY-DS has several benefits: (1) easier insertion into the biliary tract due to the tapered tip; (2) good visualization through a digital field of view of $120^{\circ}$; and (3) newly added injection and suction functions performed via a 2-port adaptor. This system thus allows the performance of EHL even for intrahepatic bile duct stones. ${ }^{10,11}$ However, if the endoscope cannot be advanced into the ampulla of Vater due to surgically altered anatomy, bile duct stone removal is also challenging. Recently, endoscopic ultrasound-guided biliary drainage (EUS-BD) such as EUSguided hepaticogastrostomy (EUS-HGS) has been developed, ${ }^{12}$ and antegrade stone removal has been reported using EUSBD technique. ${ }^{13,14}$ As an advanced and novel technique, peroral transluminal cholangioscopy (PTLC) has been also reported. ${ }^{15}$ PTLC is performed using a SPY-DS through a fistula between the intrahepatic bile duct and the stomach or intestine.

In this retrospective study, the clinical feasibility and safety of EHL for difficult bile duct stones under ERCP guidance and

Correspondence to: Takeshi Ogura

Second Department of Internal Medicine, Osaka Medical College, 2-7 Daigakuchou, Takatsuki 569-8686, Japan

Tel: +81-726831221, Fax: +81-726846532, E-mail: oguratakeshi0411@yahoo.co.jp

Received on August 3, 2017. Revised on September 13, 2017. Accepted on September 27, 2017. Published online February 8, 2018

pISSN 1976-2283 eISSN 2005-1212 https://doi.org/10.5009/gnl17352

(a) This is an Open Access article distributed under the terms of the Creative Commons Attribution Non-Commercial License (http://creativecommons.org/licenses/by-nc/4.0) which permits unrestricted non-commercial use, distribution, and reproduction in any medium, provided the original work is properly cited. 
under PTLC guidance were evaluated.

\section{MATERIALS AND METHODS}

In this pilot study, between July 2016 and July 2017, patients who were complicated with difficult bile duct stones were retrospectively enrolled. All patients provided their written, informed consent to participate before the procedure. This study was approved by the Institutional Review Board of Osaka Medical College Hospital.

\section{Technique of EHL under ERCP guidance}

Indications for EHL under ERCP guidance were as follows: (1) large or multiple bile duct stones; (2) intrahepatic bile duct stone; (3) confluence stone; and (4) the presence of a bile duct stricture. First, a duodenoscope (JF260V; Olympus Optical, Tokyo, Japan) was advanced to the ampulla of Vater, and an ERCP catheter (MTW Endoskopie, Düsseldorf, Germany) was inserted into the CBD. Next, a 0.025-inch guidewire (VisiGlide; Olympus Medical Systems, Tokyo, Japan) was placed in the biliary tract. After cholangiography, endoscopic sphincterotomy was performed. An electrohydraulic shock wave generator (Lithotron EL27; Walz Elektronik Gmbh, Herrenberg, Germany) was used to generate shock waves of increasing frequency, which were applied as a continuous sequence of discharges during EHL. A 2.4-F EHL probe was used, and EHL was performed under SPYDS guidance. To detect bile duct stones more easily, the SPY-DS was used with the mother-baby method, as previously reported. ${ }^{10}$ With the combination of operators, bile duct stones can be easily targeted.

After fragmentation, bile duct stone removal was performed using standard techniques, such as a basket or balloon catheter.
Fig. 1 shows a confluence stone, which was difficult to treat by standard technique. Cholangiography showed a defect lesion, which suggested a bile duct stone with a diameter of 25 $\mathrm{mm}$. First, the SPY-DS was inserted into the biliary tract, and the stone could be observed. Then, EHL was performed under SPY-DS guidance, and stone fragmentation could be obtained. Finally, stone removal was performed using a basket and a balloon catheter, and complete stone clearance was successfully obtained.

\section{Technique of EHL under PTLC guidance}

Indications for EHL under PTLC guidance were as follows: (1) ERCP could not be performed due to an inaccessible papilla because of surgically altered anatomy or duodenal obstruction; or (2) a large stone that could not be removed by standard techniques such as a balloon or a basket catheter. In this technique, several steps were needed. First, a fistula had to be created between the biliary tract and stomach. Second, EHL was performed through this fistula. To perform PTLC, EUS-HGS should be performed first. The echoendoscope (GF-UCT260; Olympus Optical) was inserted into the stomach, and the intrahepatic bile duct was detected. Then, the intrahepatic bile duct was punctured using a 19-gauge fine needle aspiration (Sono Tip Pro Control 19G; Medi-Globe GmbH, Achenmühle, Germany; Medico's Hirata, Osaka, Japan) needle using color Doppler to avoid puncturing any vessels. After the bile juice was aspirated, the contrast medium was injected (Fig. 2A). After obtaining the image of the biliary tract, the bile duct and stomach wall were dilated using a balloon catheter (REN biliary dilation catheter; Kaneka, Osaka, Japan) or an ERCP catheter (MTW Endoskopie) (Fig. 2B). Stent placement was performed from the intrahepatic bile duct to the stomach (EUS-HGS) using a fully covered metal
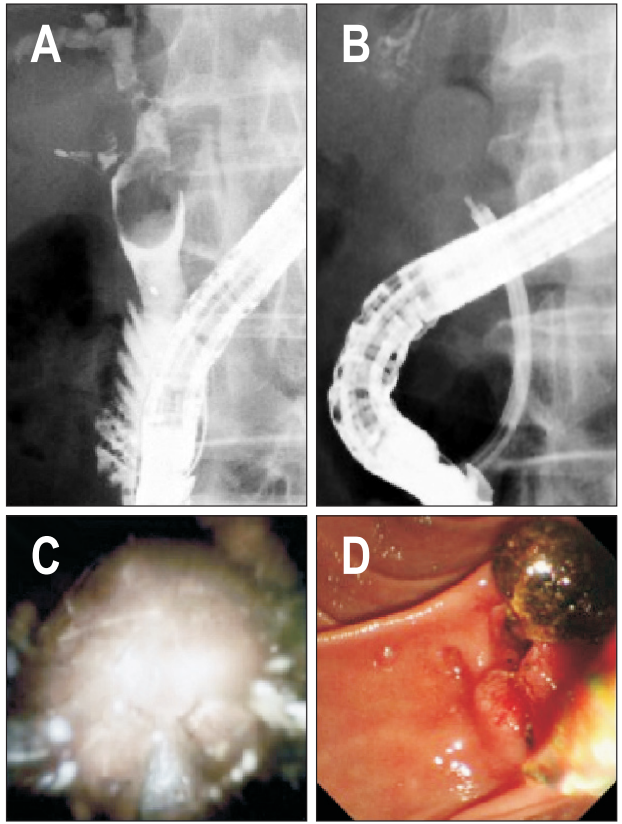

Fig. 1. (A) Cholangiography showing a large stone at the confluence of the bile duct. (B) Electrohydraulic lithotripsy image of the cholangiography. (C) Electrohydraulic lithotripsy image of the cholangioscope. (D) After the fragmentation of the bile duct stone, the stone removal is performed using a standard technique. (E) Complete stone clearance was achieved. 
stent (10 mm×100 mm, End-bare type, Niti-S Biliary Cover Stent; TaeWoong Medical, Seoul, Korea; Century Medical, Tokyo, Japan) (Fig. 2C). Although a $10 \mathrm{~cm}$-long EUS-HGS stent is useful to prevent stent migration, as previously reported, ${ }^{16,17}$ this stent has a limitation in that it is difficult to insert any devices through the stent. Therefore, if devices could not be inserted through the stent, the stent was trimmed, or the mesh of the stent was ruptured, as previously described. ${ }^{18,19}$

After the ERCP catheter was inserted into the EUS-HGS stent, the 0.025-inch guidewire (VisiGlide; Olympus Medical Systems) was advanced into the intestine across the ampulla of Vater, and the contrast medium was injected. If the CBD stone was small, antegrade stone extraction could be performed using a balloon catheter. However, if the CBD stones was large (Fig. 2A), antegrade stone extraction could not be performed using only a balloon catheter. Therefore, stone fragmentation was needed. The SPY-DS was antegradely inserted into the CBD, and the stone was visualized (Fig. 2B and C). EHL was then performed
(Fig. 3A). After stone fragmentation, the ampulla of Vater was also dilated using a balloon catheter (Fig. 3B). Lastly, antegrade stone extraction was performed. If the stones were extremely small after EHL, stone extraction was performed through the EUS-HGS stent. During this procedure, to fit the axis, the guidewire was advanced into third part of the duodenum (Fig. 3C). After stone removal, the metal stent was exchanged to a plastic stent (Type IT, 7 F, 12 cm; Gadelius Medical Co. Ltd., Tokyo, Japan) to maintain the fistula. ${ }^{20}$

\section{Definitions}

Stone size was measured by computed tomography or abdominal ultrasound. The number of bile duct stones was also counted on cholangiography. Procedure time was measured from SPY-DS insertion to removal of the duodenoscope with both ERCP and PTLC guidance. Technical success was defined as successful fragmentation using EHL under ERCP or PTLC guidance with a SPY-DS. Descriptive statistics are shown as
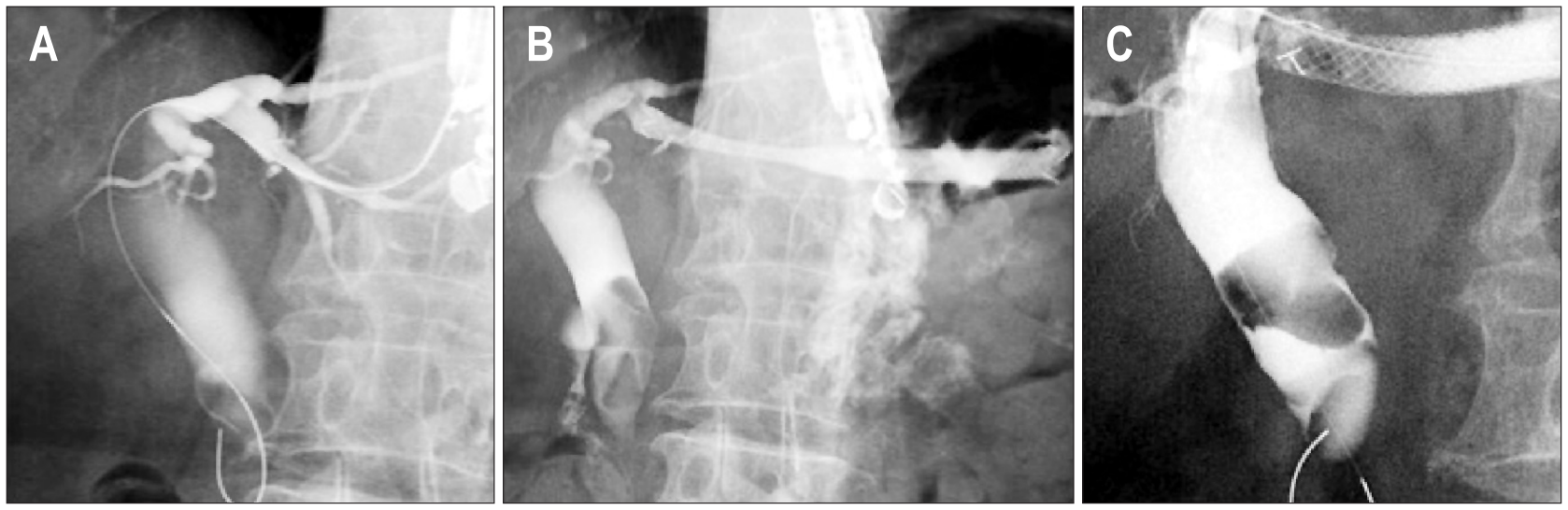

Fig. 2. (A) The intrahepatic bile duct is punctured, and the guidewire is inserted into the biliary tract. (B) Endoscopic ultrasound-guided hepaticogastrostomy is performed using a fully covered metal stent. (C) Large stones are observed during the endoscopic ultrasound-guided hepaticogastrostomy.
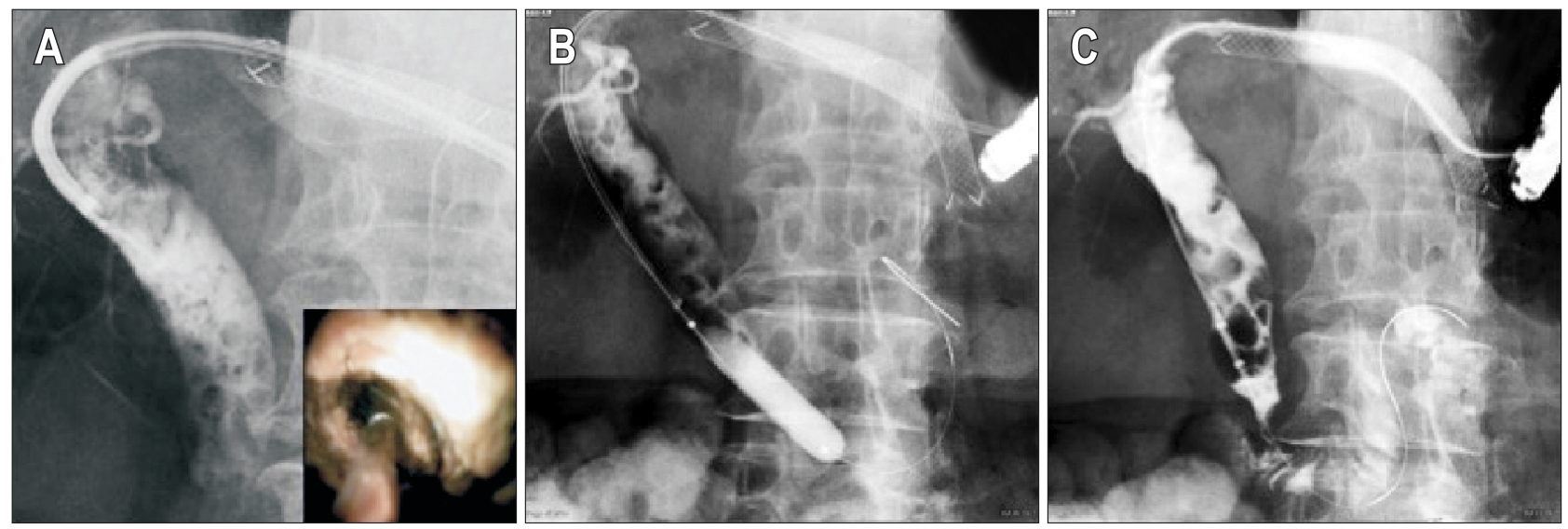

Fig. 3. (A) The digital single-operator cholangioscope (SpyGlass DS, Boston Scientific) is antegradely inserted into the common bile duct, and electrohydraulic lithotripsy is performed antegradely. (B) Balloon dilation of the ampulla of Vater is performed. (C) Stone extraction is performed antegradely. 
means \pm standard deviations or medians and range, as appropriate. Finally, adverse events were graded according to the American Society for Gastrointestinal Endoscopy lexicon's severity grading system. ${ }^{21}$

\section{RESULTS}

Table 1 shows the patients' characteristics. A total of 42 consecutive patients (27 male, 15 female) with a mean age of 77.1 years underwent EHL using the SPY-DS; 34 patients underwent EHL under ERCP guidance, and the other eight patients underwent EHL under PTLC guidance. Indications for EHL under ERCP guidance were as follows: large, multiple stones $(n=18)$, confluence stone $(n=8)$, intrahepatic bile duct stones $(n=6)$, presence of bile duct stone upstream of a biliary stricture $(n=1)$, and

Table 1. Patient Characteristics

\begin{tabular}{|c|c|}
\hline Variable & Value \\
\hline Total no. of patients & 42 \\
\hline Age, yr & $77.1 \pm 11.8$ \\
\hline Sex, male:female & $27: 15$ \\
\hline \multicolumn{2}{|l|}{ Indication of EHL under ERCP guidance $(n=34)$} \\
\hline Large, multiple stone & 18 \\
\hline Confluence stone & 8 \\
\hline Intrahepatic bile duct stone & 6 \\
\hline Left intrahepatic bile duct & 4 \\
\hline Right intrahepatic bile duct & 1 \\
\hline B3 & 1 \\
\hline $\begin{array}{l}\text { Presence of bile duct stone upstream of } \\
\text { biliary stricture }\end{array}$ & 1 \\
\hline Basket impaction & 1 \\
\hline \multicolumn{2}{|l|}{ Indication of EHL under PTLC guidance $(n=8)$} \\
\hline Surgically altered anatomy & 7 \\
\hline Malignant duodenal obstruction & 1 \\
\hline Maximum stone size, mm & $27(12-37)$ \\
\hline \multicolumn{2}{|l|}{ No. of stones } \\
\hline $1: 2: 3: 4:>4$ & $18: 6: 6: 2: 2$ \\
\hline Procedure time, min & $31(19-66)$ \\
\hline Technical success & 28/28 (100) \\
\hline No. of EHL session & $1(1-2)$ \\
\hline No. of ERCP session & $1(1-3)$ \\
\hline Rate of complete stone clearance ${ }^{*}$ & $41 / 42$ (98) \\
\hline \multicolumn{2}{|l|}{ Adverse events } \\
\hline Cholangitis & 5 \\
\hline Acute pancreatitis & 1 \\
\hline
\end{tabular}

Data are presented as number, mean \pm SD, or median (range). EHL, electrohydraulic lithotripsy; ERCP, endoscopic retrograde cholangiopancreatography; PTLC, peroral transluminal cholangioscopy. *Number/total number (\%). basket impaction $(n=1)$. On the other hand, indications for EHL under PTLC guidance were as follows: surgically altered anatomy $(n=7)$, and malignant duodenal obstruction $(n=1)$.

The maximum median stone size was $27 \mathrm{~mm}$ (range, 12 to $37 \mathrm{~mm})$, and the number of stones was as follows: $1(\mathrm{n}=18), 2$ $(n=6), 3(n=6), 4(n=2),>4(n=2)$. The median procedure time was 31 minutes (range, 19 to 66 minutes). Technical success was achieved in all patients. The median number of EHL sessions was 1 (range, 1 to 2), and that of ERCP sessions was also 1 (range, 1 to 3). The rate of complete stone clearance was $98 \%$ (41/42). One patient who failed complete bile duct stone clearance had a biliary stricture, and multiple stones were seen upstream of this stricture (Fig. 4A). To access the bile duct stone, fully covered metal stent placement was performed (Fig. 4B), and then the SPY-DS was inserted. Since a large bile duct stone was seen, EHL was also performed (Fig. 4C-E). Although many bile duct stones could be removed, complete stone clearance could not be achieved (Fig. 4F). This patient underwent surgical treatment. Finally, adverse events such as cholangitis and acute pancreatitis were seen in 14\% (6/42), which could be treated conservatively.

\section{DISCUSSION}

Approximately $10 \%$ to $15 \%$ of bile duct stones cannot be treated using standard stone removal techniques such as a basket or a balloon catheter. For such difficult stones, EHL has a clinical impact in obtaining stone fragmentation. However, EHL may also be difficult for challenging cases, such as intrahepatic bile duct stones, using a conventional cholangioscope because of limited operability due to two-way deflection and easy breakage. To overcome this problem, the SPY-DS, which has good operability with four-way deflection, and is not as fragile, has become available. However, there have not been enough reports of clinical evaluations of EHL using this scope only for the treatment of bile duct stones. Tanaka et al. ${ }^{10}$ reported a singlecenter, retrospective experience with a SPY-DS for diagnostic and therapeutic procedures. In that report, stone fragmentation was successfully performed by laser or EHL with the SPY-DS in 26 patients. Navaneethan et al. ${ }^{22}$ reported their experience with the SPY-DS in a multi-center, observational study of 105 patients. Among them, 31 patients with difficult bile duct stones underwent stone therapy by laser lithotripsy. Complete duct clearance in 1 session was achieved in 27 patients (87.1\%). As study including only bile duct stone treatment, Wong et al. ${ }^{23}$ reported laser lithotripsy with the SPY-DS under ERCP guidance. In their study, 17 patients with difficult stones were prospectively enrolled. The overall stone clearance rate was 94\% (16/17), and the rate of adverse events was $12 \%$ (2/17). This result was similar to present study, although there were differences, such as EHL and laser lithotripsy. However, the indication for EHL with the SPY-DS for intrahepatic bile duct stones may be lim- 

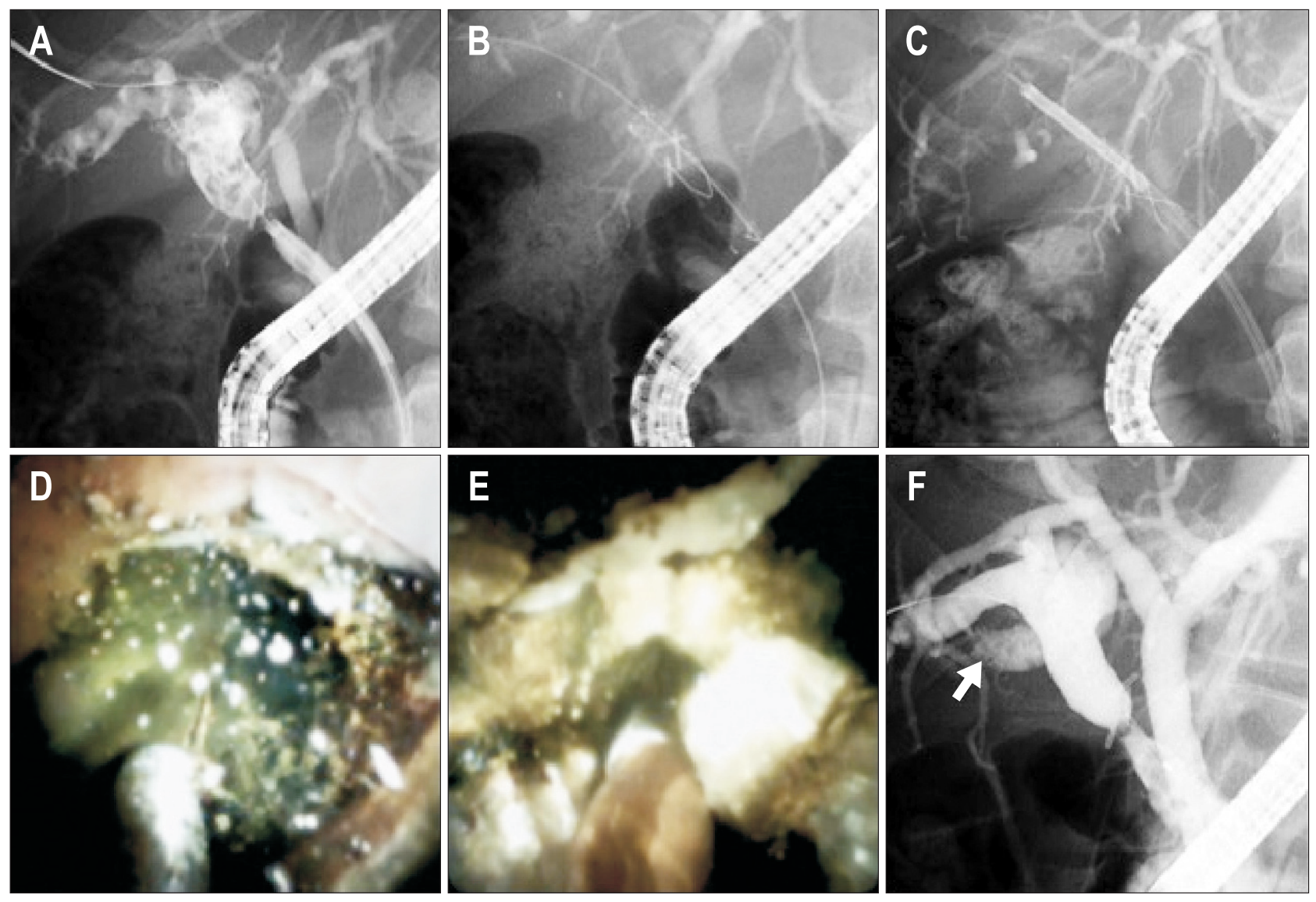

Fig. 4. (A) Bile duct stones are observed upstream of the bile duct. (B) A fully covered metal stent is inserted across the stricture site. (C) A digital single-operator cholangioscope (SpyGlass DS, Boston Scientific) is inserted into the intrahepatic bile duct through the metal stent. (D) Electrohydraulic lithotripsy is performed. (E) Fragmentation of stones is performed. (F) Complete stone clearance has failed (arrow).

ited for relatively proximal sites. Therefore, peripheral bile duct stones may not be appropriate for this approach, and this is a limitation of this method. Although the present study included a larger number of patients than these previous reports, the critical limitation of the present study is that it was retrospective. Therefore, the present results need to be confirmed by a prospective, multicenter study with a large number of patients.

In addition, the treatment of bile duct stones in patients with surgically altered anatomy is sometimes challenging. Percutaneous transhepatic drainage or a surgical approach has been used to treat such bile duct stones. Recently, single-balloon or double-balloon enteroscopy-assisted ERCP has emerged. However, although bile duct stones in patients with surgically altered anatomy may be treated using this technique, the technical success rate is less than $90 \%,{ }^{14}$ even at high-volume centers or when performed by skilled endoscopists. In the remaining 10\%, an alternative method is needed.

Recently, EUS-BD has been developed as an alternative method of biliary drainage under ERCP guidance. ${ }^{12}$ More recently, antegrade stone removal under EUS-guided biliary access has emerged. This technique has several advantages: (1) short procedure time compared with balloon enteroscopy; (2) ease of reintervention if the tract is maintained using a stent or after completion of a fistula between the bile duct and the gastrointestinal tract; and (3) cosmetic issues. ${ }^{13}$ Iwashita et al. ${ }^{14}$ reported the use of EUS-guided antegrade (EUS-AS) approach for bile duct stones in patients with surgically altered anatomy in a multicenter, retrospective, cohort study. In their study of 29 patients, 23 were successfully treated by EUS-AS. The median maximum size of the bile duct stones was $10 \mathrm{~mm}$. Adverse adverse events were as follows: mild abdominal pain $(n=2)$; bile peritonitis $(\mathrm{n}=1)$; cholecystitis $(\mathrm{n}=1)$; and elevation of serum Creactive protein $(n=1)$. EUS-AS was performed using a balloon catheter after endoscopic papillary balloon dilation in this study. However, if the size of stone is large, it might be difficult to remove the stone using only this technique. Itoi et al. ${ }^{13}$ reported the successful treatment of a large bile duct stone under EUSAS guidance. They inserted an over-the-wire type mechanical lithotripsy device into the CBD through a fistula between the intrahepatic bile duct and the jejunum. Although this technique was creative and impressive, there were several concerns. First, this technique cannot be performed for larger bile duct stones. Second, during performance of the stone fragmentation, the risk of bile juice leakage across the fistula may be increased. On the 
other hand, the present technique has several advantages. First, because of covered metal stent placement, the risk of bile leak during and after EUS-AS may be lower than with the previous technique. Second, stone removal can be performed even if the size of the bile duct stone is large. To the best of our knowledge, this is also the first report of the performance of EHL under antegrade EUS guidance.

In conclusion, EHL using a SPY-DS was technically feasible, not only with ERCP guidance, but also with PTLC guidance. A prospective, multicenter, clinical study of EHL using a SPY-DS is needed.

\section{CONFLICTS OF INTEREST}

No potential conflict of interest relevant to this article was reported.

\section{REFERENCES}

1. Yasuda I, Itoi T. Recent advances in endoscopic management of difficult bile duct stones. Dig Endosc 2013;25:376-385.

2. Trikudanathan G, Navaneethan U, Parsi MA. Endoscopic management of difficult common bile duct stones. World J Gastroenterol 2013;19:165-173.

3. Buxbaum J. Modern management of common bile duct stones. Gastrointest Endosc Clin N Am 2013;23:251-275.

4. Katanuma A, Maguchi H, Osanai M, Takahashi K. Endoscopic treatment of difficult common bile duct stones. Dig Endosc 2010;22 Suppl 1:S90-S97.

5. Ogura T, Higuchi K. A review of treatment options for bile duct stones. Expert Rev Gastroenterol Hepatol 2016;10:1271-1278.

6. Leung JW, Chung SS. Electrohydraulic lithotripsy with peroral choledochoscopy. BMJ 1989;299:595-598.

7. Binmoeller KF, Brückner M, Thonke F, Soehendra N. Treatment of difficult bile duct stones using mechanical, electrohydraulic and extracorporeal shock wave lithotripsy. Endoscopy 1993;25:201206.

8. Adamek HE, Maier M, Jakobs R, Wessbecher FR, Neuhauser T, Riemann JF. Management of retained bile duct stones: a prospective open trial comparing extracorporeal and intracorporeal lithotripsy. Gastrointest Endosc 1996;44:40-47.

9. Hui CK, Lai KC, Ng M, et al. Retained common bile duct stones: a comparison between biliary stenting and complete clearance of stones by electrohydraulic lithotripsy. Aliment Pharmacol Ther 2003;17:289-296.

10. Tanaka R, Itoi T, Honjo M, et al. New digital cholangiopancreatoscopy for diagnosis and therapy of pancreaticobiliary diseases (with videos). J Hepatobiliary Pancreat Sci 2016;23:220-226.

11. Ogura T, Imanishi M, Kurisu Y, et al. Prospective evaluation of digital single-operator cholangioscope for diagnostic and therapeutic procedures (with videos). Dig Endosc 2017;29:782-789.

12. Khashab MA, Van der Merwe S, Kunda R, et al. Prospective international multicenter study on endoscopic ultrasound-guided biliary drainage for patients with malignant distal biliary obstruction after failed endoscopic retrograde cholangiopancreatography. Endosc Int Open 2016;4:E487-E496.

13. Itoi T, Sofuni A, Tsuchiya T, Ijima M, Iwashita T. Endoscopic ultrasonography-guided transhepatic antegrade stone removal in patients with surgically altered anatomy: case series and technical review (with videos). J Hepatobiliary Pancreat Sci 2014;21:E86E93.

14. Iwashita T, Nakai Y, Hara K, Isayama H, Itoi T, Park DH. Endoscopic ultrasound-guided antegrade treatment of bile duct stone in patients with surgically altered anatomy: a multicenter retrospective cohort study. J Hepatobiliary Pancreat Sci 2016;23:227-233.

15. Ogura T, Takagi W, Kurisu Y, Higuchi K. Technical tips for peroral transluminal cholangioscopy using novel single-operator cholangioscope (with videos). J Hepatobiliary Pancreat Sci 2016;23:E25E29.

16. Ogura T, Yamamoto K, Sano T, et al. Stent length is impact factor associated with stent patency in endoscopic ultrasound-guided hepaticogastrostomy. J Gastroenterol Hepatol 2015;30:1748-1752.

17. Nakai Y, Isayama H, Yamamoto N, et al. Safety and effectiveness of a long, partially covered metal stent for endoscopic ultrasoundguided hepaticogastrostomy in patients with malignant biliary obstruction. Endoscopy 2016;48:1125-1128.

18. Ogura T, Masuda D, Takeuchi T, Fukunishi S, Higuchi K. Simplified reintervention method of EUS-guided hepaticogastrostomy stent obstruction. Gastrointest Endosc 2016;83:831.

19. Minaga K, Takenaka M, Miyata T, Ueda Y, Kitano M, Kudo M. Through-the-mesh technique after endoscopic ultrasonographyguided hepaticogastrostomy: a novel re-intervention method. Endoscopy 2016;48(S 01):E369-E370.

20. Umeda J, Itoi T, Tsuchiya T, et al. A newly designed plastic stent for EUS-guided hepaticogastrostomy: a prospective preliminary feasibility study (with videos). Gastrointest Endosc 2015;82:390396.e2.

21. Cotton PB, Eisen GM, Aabakken L, et al. A lexicon for endoscopic adverse events: report of an ASGE workshop. Gastrointest Endosc 2010;71:446-454.

22. Navaneethan U, Hasan MK, Kommaraju K, et al. Digital, singleoperator cholangiopancreatoscopy in the diagnosis and management of pancreatobiliary disorders: a multicenter clinical experience (with video). Gastrointest Endosc 2016;84:649-655.

23. Wong JC, Tang RS, Teoh AY, Sung JJ, Lau JY. Efficacy and safety of novel digital single-operator peroral cholangioscopy-guided laser lithotripsy for complicated biliary stones. Endosc Int Open 2017;5:E54-E58. 